\title{
PDT, an Adjuvant Therapy to Antibiotic Failure in Streptococcal Tonsillopharyngitis
}

\author{
Kate Cristina Blanco*, Natalia Mayumi Inada and Vanderlei Salvador Bagnato \\ São Carlos Institute of Physics, University of São Paulo, São Carlos-SP, Brazil
}

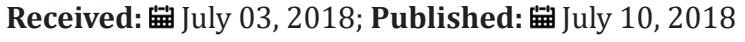

*Corresponding author: Kate Cristina Blanco, São Carlos Institute of Physics, University of São Paulo- PO Box 369, 13566-970, São

Carlos-SP, Brazil

\begin{abstract}
In the last decades, the emergence and spread of antimicrobial resistance bacteria has been viewed. Multidrug resistant bacteria are currently considered agents of worldwide emerging diseases and thus an important public health problem. Coordinated efforts to develop new therapeutic strategies are necessary. Photodynamic therapy (PDT) is a therapeutic modality based in photochemical and photophysical processes. PDT has been shown to be effective in bacterial lysis and safe in oral cavity for oral disinfection [1,2]. We report a clinical case of recurrent pharyngotonsillitis (FT) in presence of microorganisms that did not respond to antibiotic treatment. The PDT was applied in three sessions in two steps: application of pharmaceutical formulations containing $0.75 \mathrm{mg} / \mathrm{mL}$ curcumin and illumination at $450 \mathrm{~nm}$ and $6.57 \mathrm{~J} / \mathrm{cm} 2$. It was concluded that the PDT may be used as treatment in cases of antibiotic failure. In addition, periodically the PDT may be applied in case of recurrent pharyngitis.
\end{abstract}

Keywords: Antibiotic Failure; Streptococcal Tonsillopharyngitis; Photodynamic Therapy; Multidrug Resistant Bacteria

Abbreviations: PDT: Photodynamic Therapy; PS: PhotoSensitizer

\section{Background}

The patient's response to therapy may not be immediate1. It is often interpreted as a failure of medical treatment. This response should be considered due to antibiotics have the late effects[2]. Immunocompetent patients have shown a satisfactory clinical response of 48 hours in infectious processes. A patient can respond immunologically to PT maintaining its febrile state[3]. The clinical approach to these situations has included the search for a surgical alternatives such as abscess drainage and tonsil removal.Incorrect prescription of antibiotics, inadequate antibiotic action and patient immunosuppression are indicated factors as treatment failures in PT.The standard treatment for streptococcal tonsillopharyngitis has been penicillin since the 1950s. The rate of failure of penicillin has been approximately 30\%. Resistant bacterial strains are a treatment failure factor in patients with streptococcal PT.PDT occurs due to photochemical reaction which three acting factors4. The agents are: a photosensitive molecule; activating light of photosensitizer (PS) and oxygen in the environment.Two steps are necessary for occur PDT:

a) PS accumulates in the cells after its topical or systemic administration;

b) The sensitized bacterium is exposed to light at wavelength coincident to absorption spectrum of PS. The action of light and photosensitive molecule triggers two different reactions. First, there is an electronic excitation of PS by light and then two reaction mechanisms occur from the excited state. In this study, the efficacy of PDT for recurrent treatment as a adjuvant treatment after antibiotic failures was verified. We describe a clinical case of chronic PT treated with PDT.

\section{Case Representation}

The 19-year-old man, with acute pharyngitis and clinical history of antibiotic failures, was underwent PDT using curcuminoids as PS. The patient studied fulfilled all criteria of diagnosis for streptococcal PT as defined by the Centers for Disease Control. The case was admitted to hospital for an abscess drainage of infection after two cycles of penicillin with injection of 1,200,000U. After drainage of the abscess, Cefaclor 500 mg every 8 hours was prescribed. Treatment with clindamycin hydrochloride $300 \mathrm{mg} /$ dose was performed for 10 days after the episode recurrent. Unsatisfactory clinical response after 13 days of treatment was observed and Ceftriaxone $4 \mathrm{~g}$ was started once every 4 days. The tonsils surgery was indicated after no observing improvements however the patient refused. Levofloxacin $500 \mathrm{mg}$ every 12 hours was indicated for 14 days. Before the end of the treatment period, there was a recurrence of the symptoms of the disease, and Levofloxacin $500 \mathrm{mg}$ every 12 hours was indicated for 14 days. However, no improvement in clinical status was observed for seven days.

Thus, three PDT sessions during the seventh and eighth day of antibiotic treatment the PDT was performed every 8 hours. For PDT, the tonsils were sensitized with curcuminoids $0.75 \mathrm{mg} /$ 
$\mathrm{mL}$ (PDT Pharma) in a formulation (Table 1) and then performed the oropharynx illumination with blue LED $450 \mathrm{~nm}$ and $6.57 \mathrm{~J} /$ $\mathrm{cm}^{2}$.Phase A of the formulation consists of the individual weight of each component. The addition of the components was performed in a Becker under mechanical stirring and temperature of $40-60^{\circ} \mathrm{C}$ until a homogeneous and viscous mixture was formed. In Phase $\mathrm{B}$, the components were weighing and mixing of the components with pistil. In Phase $\mathrm{C}$, the citric acid was solubilized in sufficient quantity of distilled water.The patient studied presented no more clinical symptoms of disease three days after starting treatment. Clinical picture was maintained for 45 days. After that time, the patient presented a new case of infectious PT.

Table 1: Preparation of curcuminoid formulation.

\begin{tabular}{|c|c|}
\hline Component & Quantify (\%) \\
\hline \multicolumn{2}{|c|}{ Fase A } \\
\hline Agar & $15-30 \%$ \\
\hline Sugar & $0-50 \%$ \\
\hline \multicolumn{2}{|c|}{ Fase B } \\
\hline Curcuminoids & $30-50 \%$ \\
\hline Aroma & $0,1-2,0 \%$ \\
\hline Ethanol & $0,1-0,5 \%$ \\
\hline \multicolumn{2}{|c|}{ Fase C } \\
\hline Citric acid & $0,5-1 \%$ \\
\hline Distilled water & $0,1-0,5 \%$ \\
\hline
\end{tabular}

\section{Discussion}

Penicillin has brought a significant advances for treatment of infections. Several classes of antibiotics lyse bacterial walls, preventing cell proliferation[5]. In this case study, the patient might failed to respond effectively against infectionwith a higher risk of treatment failure. An unusual aspect of this case study is the failure of the treatment of intramuscular penicillin therapy[6]. Failures of antibiotic treatment have been rare with the use of parenteral penicillin. Penicillin can inhibits the development of specific antibodies and treatment failures may occur in reinfections of the same bacterial strains. Bacteria has exhibited mutations in resistance to antibiotics. The gene mutation and recombination can be transmitted during bacterial multiplication. Abusive and indiscriminate use of antibiotics by self-medication or poor indication are may favor the bacterial resistance[7]. Antimicrobial PDT and even in synergy with standard therapy may assists the destruction of antibiotic resistant bacteria. The PDT proved to be efficient in a clinical picture with several relapses of few days with failures of different antibiotics after aspiration of abscess in the tonsils. In a study carried out by Michael et al. 1994 the management of peritonsillar abscess by needle incision and drainage of abscess was evaluated and only $4.05 \%$ of recurrence of late PT episodes after abscess drainage was evaluated[8].

Cefaclor is an alternative treatment to penicillin[9]. However, same infections may be less susceptible to effects of betalactamase than penicillin. The efficacy of Cefaclor in the treatment of pharyngitis compared to penicillin has been evaluated. It was observed no statistically significant difference with patients treated with Cefaclor and penicillin. However, patients treated with penicillin were more prone to microorganisms producing beta-lactamase than patients treated with Cefaclor[9].There is no evidence of PDT cause microbial resistance which may preclude the antimicrobial action of a therapy. The mechanism action of PDT occur by transfer electrons between the photosensitive molecule in the excited triplet state and by components present in the medium generating free radicals that tend to react with oxygen in the ground state resulting in oxidized products (I). Electron transfer processes tend to be very fast due to overlapping of electronic orbitals forming an excited complex. The mechanism II occurs by transfer of energy from PS in the triplet state with generation of cytotoxic singlet oxygen. Singlet oxygen is the most cytotoxic species of the products of both mechanisms. It consists of three electronically excited states superior to molecular oxygen in the ground state. The life span of singlet oxygen depends on the nature of the medium. The cause of difference between the lifetimes is the possibility of transference of electronic energy from the singlet oxygen to some vibrational states associated with suppressor species[10].

\section{Conclusion}

In this clinical study, PDT was approached for bacterial infection of PT that presented failures during antibiotic treatment. Clinical results were satisfactory with absence of clinical symptoms of disease after $48 \mathrm{~h}$ of PDT application. There was a recurrence of the clinical case 45 days after the start of PDT. However, it shows the possibility of PDT be applied periodically in patient with PT infections after its cure to avoid recurrence processes, every 30 days, in cases of antibiotic failures.

\section{Acknowledgement}

This study was supported by São Paulo Research Foundation (FAPESP: CEPOF Grant n 13/07276-1).

\section{References}

1. Macher Jean Paul, Crocq Marc Antoine (2004) Treatment goals: Response and nonresponse. Dialogues Clin Neurosci 6(1): 83-91.

2. Langdon Amy, Crook Nathan, Dantas Gautam (2016) The effects of antibiotics on the microbiome throughout development and alternative approaches for therapeutic modulation. Genome Med 8: 39.

3. Martin Judith M (2015) The Mysteries of Streptococcal Pharyngitis. Curr Treat Options Pediatr 1(2): 180-189.

4. Allison Ron R, Moghissi Keyvan (2013) Photodynamic therapy (PDT): PDT mechanisms. Clin Endosc 46(1): 24-29.

5. Lopez R, Ronda-Lain C, Tapia A, Waks SB, Tomasz A (1976) Suppression of the lytic and bactericidal effects of cell wall inhibitory antibiotics. Antimicrob Agents Chemother 10(4): 697-706.

6. Gastanaduy Arturo S, Huwe Barbara B, Kaplan Edward L, Mckay Carolyn, Wannamaker Lewis W (1980) Failure Of Penicillin To Eradicate Group A Streptococci During An Outbreak Of Pharyngitis. Lancet 2(8193): 498502 .

7. Kimang'a AN (2012) A situational analysis of antimicrobial drug resistance in Africa: are we losing the battle?. Ethiop J Heal Sci 22(2): 135-143.

8. Wolf Michael, Even-Chen Iris, Kronenberg Jona (1994) Peritonsillar abscess: Repeated needle aspiration versus incision and drainage. Ann Otol Rhinol Laryngol 103(7): 554-557. 
9. Mc James M Cart, Renteria Antonio (1992) Treatment of pharyngitis and tonsillitis with cefprozil: Review of three multicenter trials. Clin Infect Dis 14: S224-S230.

\section{ISSN: 2574-1241}

DOI: 10.26717/BJSTR.2018.06.001377

Kate Cristina Blanco. Biomed J Sci \& Tech Res

(C) (i) This work is licensed under Creative

Submission Link: https://biomedres.us/submit-manuscript.php
10. Ogilby Peter R, Foote Christopher S (1983) Chemistry of Singlet Oxygen. 42. Effect of Solvent, Solvent Isotopic Substitution, and Temperature on

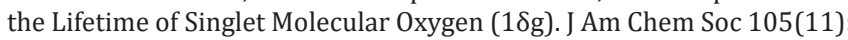
3423-3430.

BIOMEDICAL
RESEARCHES $\quad$\begin{tabular}{l} 
Assets of Publishing with us \\
\hline - Immediate, unrestricted online access
\end{tabular}

